\title{
Cross-National Differences in Psychosocial Factors of Perinatal Depression: A Systematic Review of India and Japan
}

\author{
Mizuki Takegata ${ }^{1, *}$ (D), Yukiko Ohashi ${ }^{2}$, Anisha Lazarus ${ }^{3}$ and Toshinori Kitamura ${ }^{4,5}$ \\ 1 Department of Pediatrics and Infectious Diseases, Institute of Tropical Medicine, Nagasaki University, \\ Nagasaki 852-8523, Japan \\ 2 The Faculty of Human Science Technology, Bunkyo Gakuin University, Tokyo 113-8668, Japan; \\ y-ohashi@bgu.ac.jp \\ 3 NPO Sangath, Goa 403501, India; anisha.lazarus@sangath.net \\ 4 Kitamura Institute of Mental Health Tokyo, Tokyo 151-0063, Japan; kitamura@institute-of-mental-health.jp \\ 5 Department of Psychiatry, Graduate School of Medicine, Nagoya University, Nagoya 466-8550, Japan \\ * Correspondence: mtakegata-tky@umin.ac.jp; Tel.: +81-95-819-7764
}

Academic Editor: Shervin Assari

Received: 17 October 2017; Accepted: 30 November 2017; Published: 4 December 2017

\begin{abstract}
Perinatal depression is prevalent worldwide. However, there are few available studies that discuss the different cultural factors affecting perinatal depression within Asian countries. This study aims to compare the literature regarding related factors relating to perinatal depression in India and Japan, and to synthesize the evidence common to both countries in addition to the country-specific evidence. We conducted a systematic review using several databases (CINAHL, MEDLINE, Pubmed, Ovid, SCOPUS, IndMED, and ICHUSI). Keywords were "antenatal depression" or "postpartum depression", and "India" or "Japan". Both Japanese and English language papers were reviewed. The identified evidence was compared between the two countries, as well as with non-Asian countries based on previous reports. In total, 15 articles on India and 35 on Japan were reviewed. Although several factors were shared between the two countries as well as with other non-Asian countries (vulnerable personality, being abused, age, marital conflict, and lower socio-demographic status), some differing factors were identified between India and Japan and non-Asian countries; India: poor socioeconomic status, living only with the husband, pregnancy not welcomed by the husband, a female baby, and poor relationship with in-laws; Japan: infertility treatment, conflict with work-life balance, poor relationships with biological mother or in-laws, and concerns about social relations with the other mother's friends. To conclude, involving the family and community may be important for implementing both global standardized and culture-specific interventions. In India, treatment involving the in-laws may be effective because large family structure is a significant predictor of perinatal depression. In Japan, a family/community approach involving not only the mother's family of origin but also the working environment is essential.
\end{abstract}

Keywords: cultural factors; India; Japan; perinatal depression; related factors; systematic review

\section{Introduction}

Perinatal depression occurs during pregnancy and/or within the first 12 months after delivery [1]. Symptoms such as depressed mood, loss of interest or pleasure, decreased appetite, psychomotor agitation or retardation, fatigue, feelings of guilt, insomnia, and suicidal ideation occur in various combinations [2]. Around $6.5-12.9 \%$ of women have depressive symptoms antenatally and $19.2 \%$ have postnatal depression worldwide [1]. Perinatal depression is a major health concern because it 
deteriorates not only the quality of life of mothers, but also parenting, which negatively affects the mother-infant relationship and infant development [3]. Therefore, the early detection and management of perinatal depression is critical in community settings.

Perinatal depression is prevalent in both the developing and developed countries of Asia $(3.5-63.3 \%)$ [4]. Only one review has synthesized the evidence regarding the factors related to postnatal depression in Asia. It cast light on (a) biological factors (e.g., anaemia, premenstrual cramps), (b) demographic factors (e.g., low income and primiparas), (c) interpersonal factors (e.g., intimate partner violence, unwanted pregnancy), (d) vulnerable personality (e.g., lower sense of coherence, lower self-esteem), (e) psychological factors (e.g., antenatal anxiety or depression), and (f) obstetric factors (e.g., Caesarean section and negative birth experiences) [4]. Klainin and Arthur's study (2009) also mentioned (g) cultural factors (e.g., preferred male infant), which are specific to the Asian context [4]. This result suggests to us that a culturally adapted approach should be considered in Asia. However, in the previous review there may have been insufficient evidence to examine the cultural factors of each country in Asia, due to the limited amount of English papers on the subject [4]. Hence, expanding the review of the research to also include non-English papers is an important step towards gaining broad evidence which is more focused on the local community.

Additionally, selecting two countries in Asia and discriminating between the common worldwide [5,6] and country-specific factors would be helpful. Furthermore, it would also be useful to discuss the complex social and cultural background that underlies each country's specific factors. In this review, we selected two countries: India and Japan. These countries were selected because their social and cultural aspects (such as family structures, fertility rate, and childrearing cultures) clearly differ from each other, although perinatal depression is prevalent in both India and Japan (India: antenatal depression, 6-26.3\%; postnatal depression, 7.5-45.5\%; Japan: antenatal depression, 5.6-5.8\%; postnatal depression, 5.0-21.5\%) [7-10]. India, located in South Asia, is the second most populous country in the world (over 1.2 billion people) with a total fertility rate of 2.4 [11]. India consists of multi-ethnic groups with diverse cultures and religions. India is also characterized by a joint family structure (63-86\%), where male ancestors, unmarried female offspring, and the brides of male family members usually live together under the same roof. Women, during the perinatal period, are cared for by other family members in the same household [12,13]. In contrast, Japan, located in East Asia, faces a seriously low fertility rate (1.4 in 2014) and an increase in the older age population [11]. More women are likely to get married and deliver a baby at an older age. Most families are nuclear $(60 \%)$, which has been a trend for the last three to four decades. There is a Japanese traditional custom called "Satogaeri bunben": a woman at late-term moves back to her biological parents' house for delivery in her home town, and is cared for until a few months postpartum [14]; women may migrate to their mothers' homes for the delivery period.

This study aims to compare the literature regarding related factors affecting perinatal depression in India and Japan, and to synthesize the evidence common to both countries, and other non-Asian countries, in addition to country-specific evidence.

\section{Methods}

\subsection{Search Strategies}

Relevant papers were searched for using CINAHL, MEDLINE, Pubmed, Ovid, SCOPUS, IndMED (Indian articles), and ICHUSHI (Japanese articles). ICHUSI is an online database containing articles relevant to the health sciences, including medicine, dentistry, nursing, and veterinary, only inside of Japan. First, relevant published papers were searched for using the following search terms: "antenatal depression" or "postpartum depression", and "India" or "Japan". The search was restricted to articles from within the last 20 years (from the end of November, 1996, until the end of November, 2016). Related titles and abstracts were screened according to the inclusion criteria. We conducted full text screening (Indian literature: M.T. and A.L., Japanese literature: M.T. and Y.O.). At this stage, 
additional articles were retrieved using the reference lists of the already selected published journal articles. We assessed the quality of papers when deciding the final papers to be retained (Indian literature was assessed by M.T. and A.L., Japanese literature was assessed by M.T. and Y.O.). Although we conducted a quality assessment in reference to the 'Strengthening the reporting of observational studies in epidemiology' (STROBE) statement [15], papers which were acceptable according to the minimum criteria were included in this review in order not to miss smaller evidence.

\subsection{Inclusion and Exclusion Criteria}

Studies were included if they met the following criteria (see Table S1): (a) includes in the results the investigation of factors related to perinatal depression among Indian or Japanese mothers, (b) in the case of quantitative studies, the target population was community-dwelling mothers, (c) an observational study, (d) the study is conducted in India or Japan, (e) a quantitative or qualitative study, (f) is peer-reviewed original research, (g) an article which assured the minimum acceptable quality (clear description about design, enrollment number for each observational period, setting, measurements, conducted a statistical analysis, and approved by an ethical committee). In addition to these criteria, for Indian journals, only papers written in English were scrutinized. Although their official languages are English and Hindi (the languages spoken in India are numerous), English is more recommended and used in the academic field, from basic to higher education. Researchers and clinicians usually use English rather than Hindi when publishing papers. On the other hand, it is a fact that English is still a big barrier among Japanese researchers and clinicians. Japanese is used in all levels of education. There are many peer-reviewed journals that accept Japanese written articles in the mental health field. Hence, papers written in Japanese were included. Studies recruiting Indian or Japanese mothers who lived in other countries were excluded due to consideration of possible bias from a different cultural environment.

\subsection{Review Process}

Firstly, factors relating to perinatal depression were listed for India and Japan. Secondly, common factors and country-specific factors were extracted through discussion between M.T., Y.O., and T.K. by comparing evidence between India, Japan, and an updated literature review regarding perinatal depression worldwide [5,6].

\section{Results}

\subsection{Article Extraction}

A total of 115 Indian and 756 Japanese papers were extracted from the search strategy (see Figure 1). Out of these, text screening yielded $20(17 \%)$ papers on India and 67 papers $(8 \%)$ on Japan. Finally, 15 papers (13\%) on India [10,13,16-28] and 35 papers (4\%) on Japan [7-9,14,29-59] were included in the review. Thirty-seven papers (India, $n=5$; Japan, $n=32$ ) were excluded because they did not fulfill the inclusion criteria: (a) three Indian and ten Japanese studies did not assess depression, but assessed other psychological aspects such as general health; (b) two Indian and four Japanese studies targeted a population of non-community-dwelling mothers; (c) four Japanese studies only assessed the trajectory of depression through the antenatal and postnatal periods without investigating related factors; (d) four Japanese studies did not target healthy mothers, (e) six Japanese studies were duplicates; and (f) eight Japanese studies were of poor statistical quality (unclear description of sample collection, poor sample calculation). 


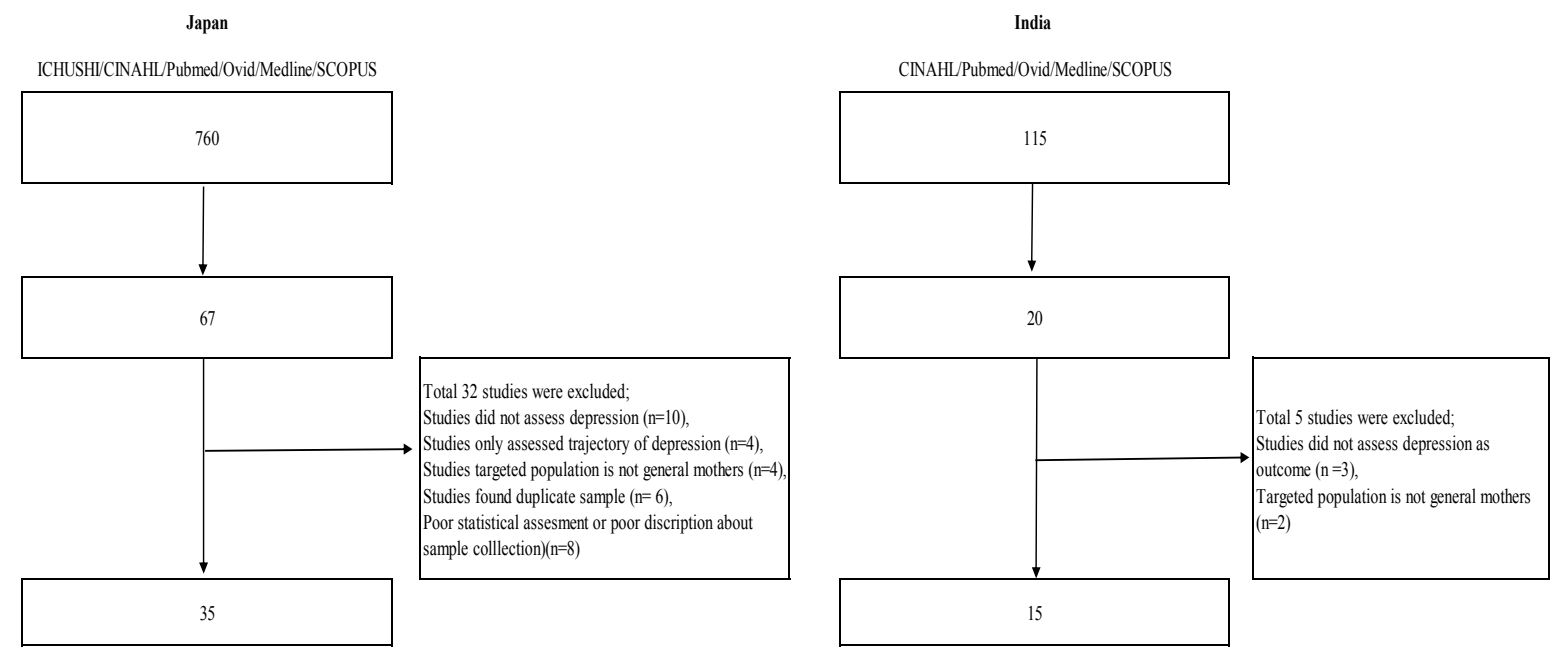

Figure 1. Related factors on perinatal depression systematic review: Flow diagram.

\subsection{Prevalence and Common Factors}

Out of 15 studies in India, 11 (73\%) were of cross-sectional design (article numbers: I1-I6, I8-I12). Three studies assessed factors relating to antenatal depression (I5, I8, I12) and others investigated those for postpartum depression. The self-questionnaires used for assessing depression in India included the Edinburgh Postnatal Depression Scale (EPDS) (I1, I3-7, I10, I11, I13, I14) [60], the Patient Health Questionnaire-9 (PHQ-9) (I2) [61], the Kessler Psychological Distress Scale (K10) (I8, I9) [62], and the Beck Depression Inventory (BDI) (I12) [63]. The Clinical Interview Schedule-Revised (CIS-R) was also used in one study (I15) [64]. Twelve out of a total of 35 Japanese studies (34\%) were of cross-sectional design (J5, J8, J9, J13, J17-19, J22, J24, J31-33). Eleven studies (31\%) investigated antenatal depression (J1, J4, J5, J6, J8, J9, J13, J17, J21, J25, J30, J34). The self-questionnaires used for assessing depression in Japan included the EPDS (J2-4, J6-8, J10-16, J18-27, J31-35), the Zung Self-Rating Depression Scale (SDS) (J28, J29, J34) [65], the Hospital Anxiety and Depression Scale (HADS) (J1) [66], and the Center for Epidemiologic Studies Depression Scale (CES-D) (J5, J9, J17) [67]. Only one study assessed depression by means of Structured Clinical Interview for DSM (SCID-DSM-IV) (J30) [68].

Among the studies using self-rating questionnaires, the prevalence of antenatal depression ranged between $16-33 \%$ in India and between $6-41 \%$ in Japan, whereas that of postpartum depression is between $7-65 \%$ in India and between $8-29 \%$ in Japan. Using structured interviews, antenatal and postnatal depression prevalence was identified as 16 and 19\%, respectively, in India; in Japan, a prevalence of $5 \%$ was identified for both antenatal and postnatal depressions.

\subsection{Common Factors}

Factors relating to perinatal depression identified across different countries were extracted through comparing the evidence between India, Japan, and non-Asian countries [5,6]. Associated factors of antenatal depression seen in India and Japan include demographic factors such as primiparas (J34), a history of mental disorder in the mother or family (I5, J4), younger age (J30), financial burden (J9, J25), and unwanted pregnancy (J9, J21, J30); vulnerable personality characteristics such as low self-directedness, high harm avoidance, and lower sense of coherence (J8, J13, J17); and interpersonal conflict including domestic violence (J1), experience of abuse (I12), and poor support from the husband or others (J13, J17).

Many factors associated with postnatal depression seen in the two countries, as well as other countries, overlapped with those of antenatal depression. They included demographic factors such as a history of mental disorder in the mother or family (I11, J27), younger age (I2, I9, I11, J14, J27, J18), and financial burden (I2, I4, I10, I13, I15, J2, J7, J9, J14); vulnerable personality characteristics such 
as a lower sense of coherence, lower self-esteem, neuroticism, and emotion-oriented coping styles (I6, J6, J10,J11, J17, J20, J23); interpersonal conflict such as domestic violence (I7), unplanned / unwanted pregnancy (I14, J9, J14, J21, J23), and poor support from husband or others (I15, J2, J3, J10, J14, J17, J18, J24, J29); and a negative life event (I15, J17). Other relevant factors which are characteristic of postnatal depression are psychological factors such as antenatal depression or anxiety (I6, J3, J12, J16), maternity blues (J26, J34), and a negative birth experience (J33).

\subsection{Factors Identified Differently between the Two Countries}

There was no factor specifically identified in each country as relating to antenatal depression. In studies in India (Table 1), poor socioeconomic status (below the poverty line, I2, I15), living only with the husband (I6), pregnancy not welcomed by the husband (I9), delivery of female baby (I1, I10, I11, I15), and poor relationships with in-laws (I15) were identified as correlating with postnatal depression. In contrast, we found the following factors correlate to postnatal depression in Japan (Table 2): infertility treatment (J21), conflict with work-life balance (J19, J23, J29), extended family household (J29, J31), poor relationships with biological parents or mother (J29, J32), concern about other relatives and in-laws (J14), and concern about social relationships with the other mother's friends (J14, J24). 
Table 1. Related factors of perinatal depression (India).

\begin{tabular}{|c|c|c|c|c|c|c|c|c|c|c|}
\hline $\begin{array}{l}\text { Article } \\
\text { Number }\end{array}$ & Author & Language & $\begin{array}{l}\text { Study } \\
\text { Design }\end{array}$ & Time Frame & Sample Size & $\begin{array}{c}\text { Outcome } \\
\text { Measurements }\end{array}$ & $\begin{array}{l}\text { Prevalence } \\
\text { (Antenatal) }\end{array}$ & $\begin{array}{l}\text { Prevalence } \\
\text { (Postnatal) }\end{array}$ & $\begin{array}{l}\text { Factors of Antenatal } \\
\text { Depression }\end{array}$ & Factors of Postnatal Depression \\
\hline I1 & $\begin{array}{c}\text { Sheela \& } \\
\text { Vankatesh (2016) } \\
{[16]} \\
\end{array}$ & English & $\begin{array}{l}\text { Cross } \\
\text { sectional }\end{array}$ & $\begin{array}{l}\text { Between } 4-7 \\
\text { weeks } \\
\text { postpartum }\end{array}$ & $\begin{array}{l}1600 \\
\text { (analysed: } \\
1600)\end{array}$ & EPDS $(\geq 13)$ & NA & $7 \%$ & NA & Delayed breastfeeding, female infant \\
\hline I2 & $\begin{array}{l}\text { Bodhare et al., } \\
\text { (2015) [17] }\end{array}$ & English & $\begin{array}{l}\text { Cross } \\
\text { sectional }\end{array}$ & $\begin{array}{l}\text { Between 6-8 } \\
\text { weeks } \\
\text { postpartum }\end{array}$ & $\begin{array}{l}287 \\
\text { (analysed: } \\
274)\end{array}$ & PHQ-9 & NA & $40 \%$ & NA & $\begin{array}{l}\text { Teenagers, poor education, poor } \\
\text { socioeconomic status, depressive } \\
\text { symptoms }\end{array}$ \\
\hline I3 & $\begin{array}{c}\text { Jain et al., (2015) } \\
\text { [18] }\end{array}$ & English & $\begin{array}{l}\text { Cross } \\
\text { sectional }\end{array}$ & $\begin{array}{c}\text { Within } 2 \\
\text { days } \\
\text { postpartum }\end{array}$ & $\begin{array}{c}1537 \\
\text { (analysed: } \\
\text { unknown) }\end{array}$ & EPDS & NA & NA & NA & Mixed breast feeding \\
\hline I4 & $\begin{array}{c}\text { Shivalli \& } \\
\text { Gururaj. (2015) } \\
\text { [13] }\end{array}$ & English & $\begin{array}{l}\text { Cross } \\
\text { sectional }\end{array}$ & $\begin{array}{l}\text { Between } 4 \\
\text { and } 6 \text { weeks } \\
\text { postpartum }\end{array}$ & $\begin{array}{l}118 \\
\text { (analysed: } \\
\text { 102) }\end{array}$ & $\operatorname{EPDS}(\geq 13)$ & NA & $65 \%$ & NA & $\begin{array}{l}\text { Poor socioeconomic status (below } \\
\text { poverty line), female baby, } \\
\text { complication of pregnancy }\end{array}$ \\
\hline I5 & $\begin{array}{l}\text { Srinivasan et al., } \\
\text { (2015) [19] }\end{array}$ & English & $\begin{array}{l}\text { Cross } \\
\text { sectional }\end{array}$ & $\begin{array}{l}\text { During } \\
\text { pregnancy }\end{array}$ & $\begin{array}{l}100 \\
\text { (analysed: } \\
100)\end{array}$ & $\operatorname{EPDS}(\geq 13)$ & NA & $26 \%$ & $\begin{array}{c}\text { First trimester, } \\
\text { idealistic distortion, } \\
\text { partner's history of } \\
\text { depression }\end{array}$ & NA \\
\hline I6 & $\begin{array}{l}\text { Johnson et al., } \\
\text { (2015) [20] }\end{array}$ & English & $\begin{array}{l}\text { Cross } \\
\text { sectional }\end{array}$ & $\begin{array}{l}\text { Within 6-8 } \\
\text { weeks } \\
\text { postpartum }\end{array}$ & $\begin{array}{l}123 \\
\text { (analysed: } \\
123 \text { ) }\end{array}$ & EPDS $(\geq 12)$ & NA & $46 \%$ & NA & $\begin{array}{l}\text { Depressed mood during pregnancy, } \\
\text { staying only with husband, lower } \\
\text { self esteem }\end{array}$ \\
\hline I7 & $\begin{array}{l}\text { Nongrum et al., } \\
\text { (2014) [21] }\end{array}$ & English & Longitudinal & $\begin{array}{l}\text { T1: During } \\
\text { pregnancy, } \\
\text { T2: After } \\
\text { delivery }\end{array}$ & $\begin{array}{l}150 \\
\text { (analysed: } \\
132 \text { ) }\end{array}$ & $\operatorname{EPDS}(\geq 12)$ & NA & NA & NA & Domestic violence \\
\hline I8 & $\begin{array}{l}\text { Lukose et al., } \\
\text { (2014) [22] }\end{array}$ & English & $\begin{array}{l}\text { Cross } \\
\text { sectional }\end{array}$ & $\begin{array}{l}12 \text { weeks of } \\
\text { pregnancy }\end{array}$ & $\begin{array}{l}366 \\
\text { (anallysed: } \\
366)\end{array}$ & $\mathrm{K}-10 \geq 6$ & $33 \%$ & NA & $\begin{array}{l}\text { Antenatal depression: } \\
\text { Having nausea, } \\
\text { vomitting, anemia. }\end{array}$ & NA \\
\hline I9 & $\begin{array}{l}\text { Prost et al., } \\
\text { (2012) [23] }\end{array}$ & English & $\begin{array}{l}\text { Cross } \\
\text { sectional }\end{array}$ & $\begin{array}{l}6 \text { weeks after } \\
\text { delivery }\end{array}$ & 5801 & $\begin{array}{c}\text { K10 scores > } \\
15\end{array}$ & NA & $12 \%$ & NA & $\begin{array}{c}\text { Younger age or older age, } \\
\text { assetqualities, health problem } \\
\text { during pregnancy, health problem } \\
\text { during delivery, health problem } \\
\text { during postnatal period, alcohol } \\
\text { consumption during pregnancy, } \\
\text { unwanted pregnancy of father }\end{array}$ \\
\hline I10 & $\begin{array}{l}\text { Dubey et al., } \\
\text { (2011) [24] }\end{array}$ & English & $\begin{array}{c}\text { Cross } \\
\text { sectional }\end{array}$ & $\begin{array}{l}\text { T1: } 34 \text { weeks } \\
\text { of } \\
\text { pregnancy, } \\
\text { T2: Within } 7 \\
\text { days } \\
\text { postpartum }\end{array}$ & $\begin{array}{l}\text { T1: } 213 \\
\text { T2: } 293 \\
\text { (analysed: } \\
\text { 506) }\end{array}$ & $\operatorname{EPDS}(\geq 10)$ & NA & $6 \%$ & NA & $\begin{array}{l}\text { Family structure, socioeconomic } \\
\text { status, marital status, female baby }\end{array}$ \\
\hline
\end{tabular}


Table 1. Cont

\begin{tabular}{|c|c|c|c|c|c|c|c|c|c|c|}
\hline $\begin{array}{l}\text { Article } \\
\text { Number }\end{array}$ & Author & Language & $\begin{array}{l}\text { Study } \\
\text { Design }\end{array}$ & Time Frame & Sample Size & $\begin{array}{l}\text { Outcome } \\
\text { Measurements }\end{array}$ & $\begin{array}{l}\text { Prevalence } \\
\text { (Antenatal) }\end{array}$ & $\begin{array}{l}\text { Prevalence } \\
\text { (Postnatal) }\end{array}$ & $\begin{array}{l}\text { Factors of Antenatal } \\
\text { Depression }\end{array}$ & Factors of Postnatal Depression \\
\hline I11 & $\begin{array}{l}\text { Savarimuthu et } \\
\text { al., (2010) [25] }\end{array}$ & English & $\begin{array}{c}\text { Cross } \\
\text { sectional }\end{array}$ & $\begin{array}{l}\text { Between } 2 \\
\text { and } 4 \text { weeks } \\
\text { postpartum }\end{array}$ & $\begin{array}{c}137 \\
\text { (analysed: } \\
137 \text { ) }\end{array}$ & EPDS ( $\geq 12)$ & NA & $26 \%$ & NA & $\begin{array}{c}\text { Teenagers or older age }(>30), \\
\text { education less than } 6 \text { years, family } \\
\text { history of depression, thought of } \\
\text { aborting current pregnancy, } \\
\text { unhappy mariage reported, alcohol } \\
\text { consumption of husband, delivery of } \\
\text { girl }\end{array}$ \\
\hline I12 & $\begin{array}{l}\text { Varma et al., } \\
(2007)[26]\end{array}$ & English & $\begin{array}{l}\text { Cross } \\
\text { sectional }\end{array}$ & $\begin{array}{l}\text { During } \\
\text { pregnancy }\end{array}$ & $\begin{array}{c}203 \\
\text { (analysed: } \\
\text { unknown) }\end{array}$ & BDI & NA & NA & $\begin{array}{l}\text { A history of sexual } \\
\text { coercion, lower life } \\
\text { satisfaction }\end{array}$ & NA \\
\hline I13 & $\begin{array}{l}\text { Rodorigues et } \\
\text { al., (2003) [27] }\end{array}$ & English & Qualitative & $\begin{array}{c}\text { 6-8 weeks } \\
\text { postpartum }\end{array}$ & $\begin{array}{l}39 \text { (analysed: } \\
\text { unknown) }\end{array}$ & $\operatorname{EPDS}(\geq 12)$ & NA & NA & NA & Unemployment \\
\hline I14 & $\begin{array}{l}\text { Patel et al., } \\
\text { (2002) [10] }\end{array}$ & English & Longitudinal & $\begin{array}{l}\text { T1: 6-8 } \\
\text { weeks } \\
\text { postpartum, } \\
\text { T2: } 6 \text { month } \\
\text { postpartum }\end{array}$ & $\begin{array}{l}252 \\
\text { (analysed: } \\
252 \text { ) }\end{array}$ & EPDS $(\geq 12)$ & NA & $\begin{array}{l}\text { T1:23\% } \\
\text { T2: } 8 \%\end{array}$ & NA & $\begin{array}{l}\text { Antenatal psychiatric morbidity, } \\
\text { unplanned pregnancy }\end{array}$ \\
\hline I15 & $\begin{array}{l}\text { Chandran et al., } \\
\text { (2002) [28] }\end{array}$ & English & Longitudinal & $\begin{array}{l}\text { T1: } 34 \text { weeks } \\
\text { of } \\
\text { pregnancy, } \\
\text { T2: } 6 \text { weeks } \\
\text { after } \\
\text { delivery }\end{array}$ & $\begin{array}{c}384 \\
\text { (analysed: } \\
354 \text { ) }\end{array}$ & $\begin{array}{c}\text { CIS-R } \\
\text { (structured } \\
\text { interview) }\end{array}$ & $\mathrm{T} 1: 16 \%$ & $\mathrm{~T} 2: 19 \%$ & NA & $\begin{array}{l}\text { Delivery of female infant, poor } \\
\text { support, lower income }(<1001 \\
\text { rupees), problems with in laws, poor } \\
\text { relationship with parents, nagative } \\
\text { life event in previous year }\end{array}$ \\
\hline
\end{tabular}


Table 2. Factors related to perinatal depression (Japan).

\begin{tabular}{|c|c|c|c|c|c|c|c|c|c|c|}
\hline $\begin{array}{l}\text { Article } \\
\text { Number }\end{array}$ & Author & Language & Design & Time Frame & Sample Size & $\begin{array}{c}\text { Outcome } \\
\text { Measurements }\end{array}$ & $\begin{array}{l}\text { Prevalence } \\
\text { (Antenatal) }\end{array}$ & $\begin{array}{l}\text { Prevalence } \\
\text { (Postnatal) }\end{array}$ & $\begin{array}{c}\text { Factors of Antenatal } \\
\text { Depression }\end{array}$ & Factors of Postnatal Depression \\
\hline $\mathrm{J} 1$ & $\begin{array}{c}\text { Kita et al., (2016) } \\
\text { [29] }\end{array}$ & English & Longitudinal & $\begin{array}{l}\text { T1: Late pregnancy } \\
\text { T2: One month } \\
\text { postpartum }\end{array}$ & $\begin{array}{c}\text { T1: } 832 \\
\text { T2: } 610 \\
\text { (analysed: } 562 \text { ) }\end{array}$ & HADS & NA & $\mathrm{NA}$ & $\begin{array}{l}\text { Antenatal intimate } \\
\text { partner violence }\end{array}$ & NA \\
\hline $\mathrm{J} 2$ & $\begin{array}{l}\text { Iwata et al., } \\
\text { (2016) [7] }\end{array}$ & English & Longitudinal & $\begin{array}{l}\text { T1: Within a few days } \\
\text { after childbirth } \\
\text { T2: One month } \\
\text { postpartum }\end{array}$ & $\begin{array}{c}\text { T1-T2: } 479 \\
\text { (analysed: } 455 \text { ) }\end{array}$ & $\operatorname{EPDS}(\geq 9)$ & NA & $\begin{array}{l}\text { T1: } 21 \% \\
\text { T2: } 21 \%\end{array}$ & NA & $\begin{array}{l}\text { Depression early postpartum, } \\
\text { financial burden, dissatisfaction with } \\
\text { appraisal support, physical burden } \\
\text { in daily life, concerns about child } \\
\text { rearing }\end{array}$ \\
\hline $\mathrm{J} 3$ & $\begin{array}{l}\text { Tachibana et al., } \\
\text { (2015) [30] }\end{array}$ & English & Longitudinal & $\begin{array}{l}\text { T1: } 20 \text { weeks of gestation } \\
\text { T2: Within a few days } \\
\text { after childbirth } \\
\text { T3: One month } \\
\text { postpartum }\end{array}$ & $\begin{array}{c}\text { T1: } 1717 \\
\text { T2: } 1335 \\
\text { T3: } 1383 \\
\text { (analysed: } 1133 \text { ) }\end{array}$ & $\operatorname{EPDS}(\geq 9)$ & NA & T1: $41 \%$ & NA & $\begin{array}{l}\text { High EPDS score during pregnancy, } \\
\text { a perceived lack of family cohesion, } \\
\text { primipara, current physical illness } \\
\text { treatment }\end{array}$ \\
\hline $\mathrm{J} 4$ & $\begin{array}{l}\text { Otake et al., } \\
\text { (2015) [8] }\end{array}$ & English & Longitudinal & $\begin{array}{c}\text { T1: } 25-35 \text { weeks of } \\
\text { pregnancy } \\
\text { T2: } 1-4 \text { months } \\
\text { postpartum } \\
\text { T3: } 6 \text { months postpartum }\end{array}$ & $\begin{array}{c}\text { T1: } 309 \\
\text { T2: } 267 \\
\text { T3: } 154 \\
\text { (analysed: } 154 \text { ) }\end{array}$ & $\operatorname{EPDS}(\geq 9)$ & $\mathrm{T} 1: 5 \%$ & $\mathrm{~T} 2: 13 \%$ & $\begin{array}{l}\text { Past depressive } \\
\text { symptoms, worrying, } \\
\text { obsessivene character }\end{array}$ & NA \\
\hline J5 & $\begin{array}{l}\text { Shirakata et al., } \\
\text { (2014) [31] }\end{array}$ & Japanese & $\begin{array}{c}\text { Cross } \\
\text { sectional }\end{array}$ & $\begin{array}{c}\text { 8-12 weeks, 23-27weeks, } \\
\text { and 35-40 gestational } \\
\text { weeks of pregnancy }\end{array}$ & $\begin{array}{c}658 \\
\text { (analysed: 352) }\end{array}$ & CES-D & NA & NA & Severe back pain & NA \\
\hline J6 & $\begin{array}{l}\text { Fukao \& } \\
\text { Kabeyama. } \\
\text { (2014) [32] }\end{array}$ & Japanese & Longitudinal & $\begin{array}{l}\text { T1: Late pregnancy } \\
\text { T2: 2-7 days postpartum } \\
\text { T3: One month } \\
\text { postpartum }\end{array}$ & $\begin{array}{l}\text { T1-T3: } 97 \\
\text { (analysed: } 97 \text { ) }\end{array}$ & $\operatorname{EPDS}(\geq 9)$ & NA & $\mathrm{NA}$ & $\begin{array}{l}\text { Lower sense of } \\
\text { coherence. }\end{array}$ & Lower sense of coherence \\
\hline $\mathrm{J} 7$ & $\underset{[33]}{\text { Amagai. (2014) }}$ & Japanese & Longitudinal & $\begin{array}{l}\text { T1: Late pregnancy } \\
\text { T2: One month } \\
\text { postpartum }\end{array}$ & $\begin{array}{c}\text { T1: } 264 \\
\text { T2: } 192 \\
\text { (analysed:153) } \\
\end{array}$ & $\operatorname{EPDS}(\geq 9)$ & NA & T2: $21 \%$ & NA & $\begin{array}{l}\text { Financial burden, non-permanent } \\
\text { position of partner }\end{array}$ \\
\hline $\mathrm{J} 8$ & $\begin{array}{l}\text { Minatani et al., } \\
\text { (2013) [34] }\end{array}$ & English & $\begin{array}{c}\text { Cross } \\
\text { sectional }\end{array}$ & Late pregnancy & $\begin{array}{c}601 \\
\text { (analysed: 601) }\end{array}$ & $\operatorname{EPDS}(\geq 9)$ & NA & NA & $\begin{array}{l}\text { Women's negative } \\
\text { response towards the } \\
\text { current pregnancy, low } \\
\text { self-directedness and } \\
\text { high harm avoidance, } \\
\text { perisistence, self } \\
\text { transcendence. }\end{array}$ & NA \\
\hline J9 & $\begin{array}{l}\text { Kinjo et al., } \\
\text { (2013) [35] }\end{array}$ & Japanese & $\begin{array}{c}\text { Cross } \\
\text { sectional }\end{array}$ & $\begin{array}{l}\text { T1: During pregnancy } \\
\text { T2: One month } \\
\text { postpartum }\end{array}$ & $\begin{array}{c}\text { T1: } 320 \\
\text { T2: } 289 \\
\text { (analysed: } 289 \text { ) } \\
\end{array}$ & CES-D & T1: $31 \%$ & T2: $33 \%$ & $\begin{array}{l}\text { Unplanned pregnancy, } \\
\text { financial burden }\end{array}$ & $\begin{array}{l}\text { Unplanned pregnancy, financial } \\
\text { burden, history of depression }\end{array}$ \\
\hline
\end{tabular}


Table 2. Cont

\begin{tabular}{|c|c|c|c|c|c|c|c|c|c|c|}
\hline $\begin{array}{l}\text { Article } \\
\text { Number }\end{array}$ & Author & Language & Design & Time Frame & Sample Size & $\begin{array}{c}\text { Outcome } \\
\text { Measurements }\end{array}$ & $\begin{array}{l}\text { Prevalence } \\
\text { (Antenatal) }\end{array}$ & $\begin{array}{l}\text { Prevalence } \\
\text { (Postnatal) }\end{array}$ & $\begin{array}{l}\text { Factors of Antenatal } \\
\text { Depression }\end{array}$ & Factors of Postnatal Depression \\
\hline $\mathrm{J} 10$ & $\begin{array}{c}\text { Sugawara \& } \\
\text { Ohira. (2013) } \\
\text { [36] }\end{array}$ & Japanese & Longitudinal & $\begin{array}{l}\text { T1: Middle pregnancy } \\
\text { T2: Late pregnancy } \\
\text { T3: One month } \\
\text { postpartum }\end{array}$ & $\begin{array}{l}\text { T1-T3: } 80 \\
\text { (analysed: } 72 \text { ) }\end{array}$ & $\operatorname{EPDS}(\geq 9)$ & $\begin{array}{l}\text { T1: } 16 \% \\
\text { T2: } 12 \%\end{array}$ & T3: $16 \%$ & NA & $\begin{array}{l}\text { Lower sense of coherence, poor } \\
\text { social support }\end{array}$ \\
\hline $\mathrm{J} 11$ & $\begin{array}{l}\text { Urayama et al., } \\
\text { (2013) [37] }\end{array}$ & Japanese & Longitudinal & $\begin{array}{l}\text { T1: Within five days } \\
\text { postpartum } \\
\text { T2: One month } \\
\text { postpartum }\end{array}$ & $\begin{array}{c}\text { T1: } 101 \\
\text { T2: } 101 \\
\text { (analysed: 100) }\end{array}$ & $\operatorname{EPDS}(\geq 9)$ & NA & $\begin{array}{l}\text { T1: } 33 \% \\
\text { T2: } 18 \%\end{array}$ & NA & Lower self efficacy, lower self esteem \\
\hline $\mathrm{J} 12$ & $\begin{array}{l}\text { Sugishita \& } \\
\text { Kamibeppu. } \\
\text { (2013) [38] }\end{array}$ & Japanese & Longitudinal & $\begin{array}{l}\text { T1: Late pregnancy } \\
\text { T2: One month } \\
\text { postpartum }\end{array}$ & $\begin{array}{c}\text { T1: } 161 \\
\text { T2: } 121 \\
\text { (analysed:121) }\end{array}$ & $\operatorname{EPDS}(\geq 9)$ & T1: $14 \%$ & T2: $19 \%$ & NA & Antenatal depression \\
\hline $\mathrm{J} 13$ & $\begin{array}{l}\text { Miyamoto. } \\
\text { (2012) [39] }\end{array}$ & Japanese & $\begin{array}{c}\text { Cross } \\
\text { sectional }\end{array}$ & During pregnancy & $\begin{array}{c}128 \\
\text { (analysed: } 128 \text { ) }\end{array}$ & $\operatorname{EPDS}(\geq 9)$ & $15 \%$ & NA & $\begin{array}{l}\text { Depressive schema, } \\
\text { Intrauterine growth } \\
\text { restriction (IUGR), poor } \\
\text { relationship with } \\
\text { partner }\end{array}$ & NA \\
\hline $\mathrm{J} 14$ & $\begin{array}{c}\text { Nagasaka \& } \\
\text { Sano. (2012) [40] }\end{array}$ & Japanese & Longitudinal & $\begin{array}{l}\text { T1: Early pregnancy } \\
\text { T2: One month } \\
\text { postpartum } \\
\text { T3: Four month } \\
\text { postpartum }\end{array}$ & $\begin{array}{c}\text { T1: } 3080 \\
\text { T2: } 2420 \\
\text { T3: } 2420 \\
\text { (analysed: } 2420 \text { ) }\end{array}$ & $\operatorname{EPDS}(\geq 9)$ & NA & $\begin{array}{l}\text { T2: } 13 \% \\
\text { T3: } 12 \%\end{array}$ & NA & $\begin{array}{l}\text { T4: teenager, primiparas, unwanted } \\
\text { pregnancy, poor support from } \\
\text { husband, smoking, history of mental } \\
\text { illness, concern about childrearing, } \\
\text { economic concern, concern about } \\
\text { social relations with other mothers, } \\
\text { concern about other relative }\end{array}$ \\
\hline $\mathrm{J} 15$ & $\begin{array}{l}\text { Hayakawa et al., } \\
\quad(2012)[41]\end{array}$ & English & Longitudinal & $\begin{array}{l}\text { T1: Early postpartum } \\
\text { T2: Late pregnancy } \\
\text { T3: One month } \\
\text { postpartum }\end{array}$ & $\begin{array}{c}\text { T1-T3: 467 } \\
\text { (analysed: } 448 \text { ) }\end{array}$ & $\operatorname{EPDS}(\geq 9)$ & T2: $13.2 \%$ & NA & NA & Lower maternal care (PBI) \\
\hline $\mathrm{J} 16$ & $\begin{array}{l}\text { Kokubu et al., } \\
(2012)[42]\end{array}$ & English & Longitudinal & $\begin{array}{l}\text { T1: Late pregnancy } \\
\text { T2: Early postpartum } \\
\text { T3: One month } \\
\text { postpartum }\end{array}$ & $\begin{array}{l}\text { T1-T3: } 109 \\
\text { (analysed: } 99 \text { ) }\end{array}$ & $\operatorname{EPDS}(\geq 9)$ & NA & NA & NA & Antenatal anxiety \\
\hline $\mathrm{J} 17$ & $\begin{array}{l}\text { Kinjo et al., } \\
\text { (2011) [43] }\end{array}$ & Japanese & $\begin{array}{l}\text { Cross } \\
\text { sectional }\end{array}$ & $\begin{array}{l}\text { T1: During pregnancy } \\
\text { T2: One month } \\
\text { postpartum }\end{array}$ & $\begin{array}{c}\text { T1: } 158 \\
\text { T2: } 164 \\
\text { (analysed:164) }\end{array}$ & CES-D & T1: $30.4 \%$ & T2: $24.4 \%$ & $\begin{array}{l}\text { Perceived stress, lower } \\
\text { self esteem, poor social } \\
\text { support }\end{array}$ & $\begin{array}{l}\text { Perceived stress, lower self esteem, } \\
\text { poor social support }\end{array}$ \\
\hline
\end{tabular}


Table 2. Cont

\begin{tabular}{|c|c|c|c|c|c|c|c|c|c|c|}
\hline $\begin{array}{l}\text { Article } \\
\text { Number }\end{array}$ & Author & Language & Design & Time Frame & Sample Size & $\begin{array}{c}\text { Outcome } \\
\text { Measurements }\end{array}$ & $\begin{array}{l}\text { Prevalence } \\
\text { (Antenatal) }\end{array}$ & $\begin{array}{l}\text { Prevalence } \\
\text { (Postnatal) }\end{array}$ & $\begin{array}{l}\text { Factors of Antenatal } \\
\text { Depression }\end{array}$ & Factors of Postnatal Depression \\
\hline $\mathrm{J} 18$ & $\begin{array}{l}\text { Mori et al., } \\
\text { (2011) [44] }\end{array}$ & English & $\begin{array}{c}\text { Cross } \\
\text { sectional }\end{array}$ & $\begin{array}{l}\text { Within } 4 \text { weeks } \\
\text { postpartum }\end{array}$ & $\begin{array}{c}675 \\
\text { (analysed: 675) }\end{array}$ & $\operatorname{EPDS}(\geq 9)$ & NA & $\mathrm{T} 1: 11 \%$ & NA & $\begin{array}{c}\text { Early onset depression (within } 4 \\
\text { weeks): Lack of emotional support, } \\
\text { psychiatric history } \\
\text { Late onset depression (5-12 weeks): } \\
\text { younger age(less than } 25 \text { years old), } \\
\text { older age (older than } 36 \text { years old), } \\
\text { history of depression }\end{array}$ \\
\hline $\mathrm{J} 19$ & $\begin{array}{l}\text { Miyake et al., } \\
(2011)[45]\end{array}$ & English & $\begin{array}{c}\text { Cross } \\
\text { sectional }\end{array}$ & $\begin{array}{l}\text { Between } 3 \text { and } 4 \text { months } \\
\text { postpartum }\end{array}$ & $\begin{array}{c}771 \\
\text { (analysed: } 771 \text { ) }\end{array}$ & $\operatorname{EPDS}(\geq 9)$ & NA & $14 \%$ & NA & Fulltime workers \\
\hline $\mathrm{J} 20$ & $\begin{array}{l}\text { Kikuchi et al., } \\
\text { (2010) [46] }\end{array}$ & Japanese & Longitudinal & $\begin{array}{l}\text { T1: Middle pregnancy } \\
\text { T2: One month } \\
\text { postpartum }\end{array}$ & $\begin{array}{l}\text { T1: } 243 \\
\text { T2: } 163 \\
\text { (analysed: } 113 \text { ) }\end{array}$ & $\operatorname{EPDS}(\geq 9)$ & NA & $15 \%$ & NA & $\begin{array}{l}\text { Personality (Neuroticism, low } \\
\text { extravert, low agreeableness, low } \\
\text { conciousness, emotional oriented } \\
\text { coping style), high protection from } \\
\text { father }\end{array}$ \\
\hline $\mathrm{J} 21$ & $\begin{array}{l}\text { Iwamoto et al., } \\
\text { (2010) [47] }\end{array}$ & Japanese & Longitudinal & $\begin{array}{l}\text { T1: Late pregnancy } \\
\text { T2: } 5 \text { days postpartum } \\
\text { T3: One month } \\
\text { postpartum } \\
\text { T4: } 4 \text { months postpartum } \\
\end{array}$ & $\begin{array}{c}590 \\
\text { (analysed: 590) }\end{array}$ & $\operatorname{EPDS}(\geq 9)$ & NA & NA & Unplanned pregnancy & $\begin{array}{l}\text { Unplanned pregnancy, pregnancy by } \\
\text { infertility treatment }\end{array}$ \\
\hline $\mathrm{J} 22$ & $\begin{array}{c}\text { Arai \& } \\
\text { Takahashi. } \\
\text { (2009) [48] }\end{array}$ & Japanese & $\begin{array}{c}\text { Cross } \\
\text { sectional }\end{array}$ & One month postpartum & $\begin{array}{c}283 \\
\text { (analysed: 149) }\end{array}$ & $\operatorname{EPDS}(\geq 9)$ & NA & $21 \%$ & NA & $\begin{array}{l}\text { Overall functioning, lower affective } \\
\text { responsiveness, lower affective } \\
\text { involvement }\end{array}$ \\
\hline $\mathrm{J} 23$ & $\begin{array}{l}\text { Ando \& Muto. } \\
\text { (2009) [49] }\end{array}$ & Japanese & Longitudinal & $\begin{array}{l}\text { T1: During pregnancy, } \\
\text { T2: } 5 \text { weeks postpartum, } \\
\text { T3: } 3 \text { months postpartum, } \\
\text { T4: } 6 \text { months postpartum, } \\
\text { T5: } 1 \text { year after delivery }\end{array}$ & $\begin{array}{c}\text { T1-3: 522 } \\
\text { (analysed: 407) }\end{array}$ & $\operatorname{EPDS}(\geq 9)$ & NA & NA & NA & $\begin{array}{l}\text { Unwanted pregnancy, less delighted } \\
\text { with fetal movement, morning } \\
\text { sickness, conflict with work life } \\
\text { balance, poor marital relationship, } \\
\text { self absorption, lower self esteem, } \\
\text { low attachment with others }\end{array}$ \\
\hline $\mathrm{J} 24$ & $\begin{array}{l}\text { Satoh et al., } \\
\text { (2009) [50] }\end{array}$ & English & $\begin{array}{c}\text { Cross } \\
\text { sectional }\end{array}$ & 4 months postpartum & $\begin{array}{c}169 \\
\text { (analysed: } 169)\end{array}$ & $\operatorname{EPDS}(\geq 9)$ & NA & $23 \%$ & NA & $\begin{array}{c}\text { General health abnormality, poor } \\
\text { sociability, abnormality, worry about } \\
\text { baby care, poor cooperation of the } \\
\text { husband }\end{array}$ \\
\hline $\mathrm{J} 25$ & $\begin{array}{l}\text { Kanazawa et al., } \\
(2008)[51]\end{array}$ & Japanese & Longitudinal & $\begin{array}{l}\text { T1: During pregnancy, } \\
\text { T2: Within a few days } \\
\text { after delivery, } \\
\text { T3: One month } \\
\text { postpartum }\end{array}$ & $\begin{array}{c}112 \\
\text { (analysed: 111) }\end{array}$ & $\operatorname{EPDS}(\geq 9)$ & NA & $14 \%$ & $\begin{array}{c}\text { financial burden, } \\
\text { smoking or alcohol } \\
\text { comsumption durin } \\
\text { pregnancy. }\end{array}$ & $\begin{array}{l}\text { Lower confidence of child rearing, } \\
\text { perceived stress with childrearing, } \\
\text { concern about baby's condition }\end{array}$ \\
\hline
\end{tabular}


Table 2. Cont.

\begin{tabular}{|c|c|c|c|c|c|c|c|c|c|c|}
\hline $\begin{array}{l}\text { Article } \\
\text { Number }\end{array}$ & Author & Language & Design & Time Frame & Sample Size & $\begin{array}{l}\text { Outcome } \\
\text { Measurements }\end{array}$ & $\begin{array}{l}\text { Prevalence } \\
\text { (Antenatal) }\end{array}$ & $\begin{array}{l}\text { Prevalence } \\
\text { (Postnatal) }\end{array}$ & $\begin{array}{l}\text { Factors of Antenatal } \\
\text { Depression }\end{array}$ & Factors of Postnatal Depression \\
\hline $\mathrm{J} 26$ & $\begin{array}{l}\text { Endo et al., } \\
\text { (2008) [52] }\end{array}$ & Japanese & Longitudinal & $\begin{array}{l}\text { T1: 5th day postpartum } \\
\text { T2: One month } \\
\text { postpartum }\end{array}$ & $\begin{array}{c}57 \\
\text { (analysed: } 57 \text { ) }\end{array}$ & $\operatorname{EPDS}(\geq 9)$ & NA & $30 \%$ & NA & Older age, maternity blues \\
\hline $\mathrm{J} 27$ & $\begin{array}{l}\text { Mitamura.(2008) } \\
{[53]}\end{array}$ & Japanese & Longitudinal & $\begin{array}{l}\text { T1: Within a few days } \\
\text { after delivery } \\
\text { T2: One month } \\
\text { postpartum }\end{array}$ & $\begin{array}{c}\text { T1: } 549 \\
\text { T2: } 503 \\
\text { (analysed: } 503 \text { ) }\end{array}$ & $\operatorname{EPDS}(\geq 9)$ & NA & $8 \%$ & NA & $\begin{array}{l}\text { Teenagers, divorce during pregnancy } \\
\text { or after delivery, history of mental } \\
\text { illness, maternity blues }\end{array}$ \\
\hline $\mathrm{J} 28$ & $\begin{array}{l}\text { Sekizuka et al., } \\
\text { (2007) [54] }\end{array}$ & Japanese & Longitudinal & $\begin{array}{l}\text { T1: Late pregnancy, } \\
\text { T2: Between } 3-5 \text { days } \\
\text { after delivery }\end{array}$ & $\begin{array}{l}\text { T1-2: } 54 \\
\text { (analysed: } 54 \text { ) }\end{array}$ & SDS & NA & NA & NA & $\begin{array}{l}\text { Lower sense of coherence, lower } \\
\text { satisfaction of delivery }\end{array}$ \\
\hline $\mathrm{J} 29$ & $\begin{array}{c}\text { Sato et al., (2006) } \\
{[55]}\end{array}$ & Japanese & Longitudinal & $\begin{array}{l}\text { T1: Late pregnancy, } \\
\text { T2: 5th day postpartum, } \\
\text { T3: One month } \\
\text { postpartum, } \\
\text { T4: Three month } \\
\text { postpartum }\end{array}$ & $\begin{array}{l}\text { T1-T3: } 58 \\
\text { (analysed: } 58 \text { ) }\end{array}$ & SDS & NA & NA & NA & $\begin{array}{l}\text { T4: Multipara, Living with extended } \\
\text { family, perceived negative } \\
\text { relationship with their husband, } \\
\text { biological mother, difficulty in baby's } \\
\text { treatment, lack of satisfaction from } \\
\text { husband's support and mother's } \\
\text { support, unbalanced working model, } \\
\text { negative feeling toward the baby, } \\
\text { negative feeling toword the baby, } \\
\text { low maternal attachment, anxiety } \\
\text { regarding children }\end{array}$ \\
\hline $\mathrm{J} 30$ & $\begin{array}{l}\text { Kitamura et al., } \\
\quad(2006)[9]\end{array}$ & English & Longitudinal & $\begin{array}{l}\text { T1: Late pregnancy } \\
\text { T2: One month } \\
\text { T3: Three months } \\
\text { postpartum }\end{array}$ & T1-T3: 303 & SCID & T1: $5 \%$ & $\mathrm{~T} 2: 5 \%$ & $\begin{array}{l}\text { Younger age, negative } \\
\text { attitude towards the } \\
\text { current pregnancy }\end{array}$ & $\begin{array}{l}\text { Poor accomodation, disatisfaction } \\
\text { with child sex }\end{array}$ \\
\hline $\mathrm{J} 31$ & $\begin{array}{l}\text { Ninagawa et al., } \\
\text { (2005) [56] }\end{array}$ & Japanese & $\begin{array}{c}\text { Cross } \\
\text { sectional }\end{array}$ & 2 month postpartum & $\begin{array}{c}332 \\
\text { (analysed: 289) }\end{array}$ & $\operatorname{EPDS}(\geq 9)$ & NA & $16 \%$ & NA & Extended family household \\
\hline $\mathrm{J} 32$ & $\begin{array}{l}\text { Suzumiya et al., } \\
\quad(2004)[14]\end{array}$ & Japanese & $\begin{array}{l}\text { Cross } \\
\text { sectional }\end{array}$ & $\begin{array}{l}\text { Within } 3 \text { months } \\
\text { postpartum }\end{array}$ & $\begin{array}{c}3370 \\
\text { (analysed: 3370) }\end{array}$ & $\operatorname{EPDS}(\geq 9)$ & NA & $14 \%$ & NA & $\begin{array}{l}\text { Pregnancy anomaly, experience of } \\
\text { seeing psychiatrist or counsellors, } \\
\text { Previous still birth, miscarriage, } \\
\text { experience that family has passed } \\
\text { away, poor social support from } \\
\text { biological parents, poor social } \\
\text { support from others, satisfied with } \\
\text { regidencial place, financial burden, } \\
\text { illness of infant }\end{array}$ \\
\hline $\mathrm{J} 33$ & $\begin{array}{c}\text { Tokiwa.(2003) } \\
\text { [57] }\end{array}$ & Japanese & $\begin{array}{l}\text { Cross } \\
\text { sectional }\end{array}$ & $\begin{array}{l}\text { Within seven days } \\
\text { pospartum }\end{array}$ & $\begin{array}{c}1500 \\
\text { (analysed: 932) }\end{array}$ & $\operatorname{EPDS}(\geq 9)$ & NA & NA & NA & $\begin{array}{l}\text { Negative birth experience, } \\
\text { dissatisfaction with medical staffs, } \\
\text { younger age, higher anxiety }\end{array}$ \\
\hline
\end{tabular}


Table 2. Cont

\begin{tabular}{|c|c|c|c|c|c|c|c|c|c|c|}
\hline $\begin{array}{l}\text { Article } \\
\text { Number }\end{array}$ & Author & Language & Design & Time Frame & Sample Size & $\begin{array}{c}\text { Outcome } \\
\text { Measurements }\end{array}$ & $\begin{array}{l}\text { Prevalence } \\
\text { (Antenatal) }\end{array}$ & $\begin{array}{l}\text { Prevalence } \\
\text { (Postnatal) }\end{array}$ & $\begin{array}{l}\text { Factors of Antenatal } \\
\text { Depression }\end{array}$ & Factors of Postnatal Depression \\
\hline $\mathrm{J} 34$ & $\begin{array}{l}\text { Iwatani et al., } \\
\text { (2001) [58] }\end{array}$ & Japanese & Longitudinal & $\begin{array}{l}\text { T1: Early pregnancy } \\
\text { T2: Five days postpartum } \\
\text { T3: One month } \\
\text { postpartum }\end{array}$ & $\begin{array}{c}\text { T1-T2: 252 } \\
\text { (analysed: 252) }\end{array}$ & $\begin{array}{l}\text { SDS (T1 and } \\
\text { T2) } \\
\text { EPDS (T2) }\end{array}$ & $\mathrm{T} 1: 31 \%$ & T2: $13 \%$ & Primiparas & Maternity blues \\
\hline $\mathrm{J} 35$ & $\begin{array}{l}\text { Tamaki et al., } \\
\text { (1997) [59] }\end{array}$ & English & Longitudinal & $\begin{array}{l}\text { T1: One month } \\
\text { postpartum, } \\
\text { T2: Three months } \\
\text { postpartum } \\
\text { T3: Four months } \\
\text { postpartum }\end{array}$ & $\begin{array}{l}\text { T1: } 672 \\
\text { T2: } 1096 \\
\text { T3: } 822 \\
\text { T4: } 913 \\
\text { (analysed: } 672 \text { ) }\end{array}$ & $\operatorname{EPDS}(\geq 9)$ & NA & $\begin{array}{c}\text { T1: } 18 \% \\
\text { T2: } 12.1 \% \\
\text { T3: } 6.7 \%\end{array}$ & NA & $\begin{array}{l}\text { T1: primiparas, worry about child } \\
\text { care, higher anxiety, } \\
\text { T2: negative life events, worry about } \\
\text { childcare, higher anxiety }\end{array}$ \\
\hline
\end{tabular}

Note: NA (Not assessed). 


\section{Discussion}

This literature review compared evidence regarding perinatal depression in India and Japan. As well as common factors, some country-specific factors were identified. Whereas interpersonal factors relating to family characteristics strongly influence the onset of perinatal depression in Indian women, conflict within the work environment as well as with family members was a serious issue in Japanese women.

\subsection{The Prevalence of Antenatal and Postnatal Depression}

The prevalence in India ranged more widely (antenatal depression, 16-33\%; postnatal depression, $7-65 \%)$ than other low-middle income countries $[4,69,70]$ : many studies showed a prevalence of more than 20\%, while Malaysia and Nepal had lower prevalences (4\%) and Pakistan and Turkey had higher prevalences (Pakistan, 36-63\%; Turkey, 14-50\%). This may reflect the diverse cultures, settings, and ethnicites in India. The rate in Japan (antenatal depression, 6-41\%; postnatal depression, 8-29\%) is similar to that of other high-income countries $[1,4,5]$. Antenatal depression rates might be higher than other countries. However, the generalizability of the findings should be treated cautiously because it may be partly due to the various adopted cutoff points between the two countries and/or scales of depression used [60-68].

It is of note that most studies did not use a structured diagnostic interview such as SCID. Their diagnosis mainly relied on the results of questionnaires such as the EPDS. Cut-off points for the EPDS varied (11/12 vs. 12/13) depending on researchers. Some studies included in our review followed Patel's study [10] using a cut-off score of 11/12. Although Fernandes et al. (2011), recommended cut off score of 12/13 in assessing antenatal depression in the rural setting [71], some studies assessed both antenatal/postnatal depression using the same cut off score. In Japan, there exists only one study examining the appropriate cut-off point for the EPDS using a combination of cases of major and minor depression [72]. This study proposed 8/9 as the best cut-off point for the EPDS to identify cases with major or minor depression. We must pay attention to the varying nature of appropriate cut-off points of questionnaires used over the perinatal period [73].

\subsection{Common Factors}

Several factors were associated with both antenatal and postnatal depression. Interpersonal conflict with partner-which includes domestic violence, experience of abuse, unwanted pregnancy, and poor support-is a major contributing factor to perinatal depression. Perinatal depression is a transitional period for both the husband and wife, which occurs as they gain parenthood, reform their relationship, adapt to a new environment, and welcome new family members. A crisis is more likely to occur in the process of transformation. In addition to this, being exposed to either traumatic events or abuse by the partner lowers an individual's coping ability, which leads to being vulnerable to stressful events. While these factors linked to the onset of depression are probably universal, the results suggest that healthcare providers should be aware of both pregnancy planning and marital relationship-related factors when providing care.

\subsection{Interpersonal Characteristics in the Cultural Contexts of Asian Countries}

As several studies in this review demonstrated significant associations between having poor relationships with family, relatives, and others (e.g., mother's friends, colleagues) and the onset of depressive symptoms during the perinatal period both in India and Japan, interpersonal relationships may be a key determinant in Asian societies. Whereas "individualism" is more valued in Western countries, "interdependence" is often described as an important value system in Asian cultures [74]. Kitayama et al. (1997), noted that "individuals (originally from western countries) are motivated to discover and develop positively valued internal attributes of the self, express them in public, and may develop a variety of social psychological processes that enable them to maintain and increase their 
self-esteem" [74]. This suggests that the satisfaction and well being of individuals are derived from having personal control over stressful situations in western countries. On the other hand, individuals in Asian countries are more required to adjust themselves into the society (organization). The spirit of cooperation, affection, and self-sacrifice, which are regarded as social virtues, are developed in individuals who grow through interactions with family, the work-place, and community members. Such virtues would be helpful to strengthen ties in family and work environments and are a great resource for mothers. However, we assume some women tend not to express their own needs and ask for help (self-sacrifice) because they recognize they should play their role (as a wife, as a colleague, and as a caregiver) adjusted to their family, work-place, and community (cooperation). Considering the the antenatal and postnatal period is challenging to mothers because it is also a dynamic period of transition to motherhood, the psychological distress of mothers within interpersonal relationships may be increased.

\subsection{Interpersonal Factors in India and Japan}

Although interpersonal factors such as poor relationships with family members and others are commonly identified in the two countries, social and cultural backgrounds may differ. Compared with Japan, India is characterized by strong ties within family members. A clear-cut gender role attitude exists in Indian society, where men work outside the home and women are responsible for doing household chores and caring for babies [75]. This may have pros and cons. Living in a large family, women are able to have more opportunities to share their experiences during the perinatal period. They can gain knowledge or skills for childrearing from other family members. This is also a source of economic security. There is also strong merit in mothers receiving assurance in several decisions regarding childrearing through consultations with family members. Having a good relationship with female family members would be important for the adaptive transition to motherhood. On the other hand, negative relationships between family members are likely to have adverse effects on the process of pregnancy, childbirth, and the postnatal period. Too much of an unequal power balance between the mother-in-law and bride (conflict with mother-in-law) results in little opportunity for the bride to express her feelings, leading to the onset of depression. If a woman lives only with her husband, it may be perceived as a serious lack of social support. Similarly, the situation where the woman is the sole decision maker about pregnancy and delivery is extremely stressful for an Indian woman.

In terms of Japan, the factor 'extended family structure' seems to be similar to the factor 'poor relationship with mother-in-law' identified in India, however, we assume these are different situations. According to Ninagawa [56], it was assumed that because of the increased workload (including house-chores), there may increased stress for mothers living in large families. The fact that 'conflict with biological mother' was identified as a significant contributor to perinatal depression is of interest as this may reflect a modern Japanese society where the majority of families are nuclear and relationships with other relatives are relatively scarce [76]. More mothers expect to receive emotional and practical support from their biological mothers as well as husbands. Regardless of 'Satogaeri bunben', biological mothers are key persons from whom to inherit related knowledge and skills regarding delivery and childrearing in the Japanese tradition $[49,77]$. However, there are some conflicts that occur between biological mothers and daughters that arise from differences between the traditional and modern childrearing styles.

It is of interest that mothers who were concerned about establishing social relationships with other mothers during pregnancy and after childbirth were more likely to manifest depression after delivery [40]. Pessimistic images regarding getting involved in new social relationships with other "mother-friends" after childbirth are a common topic of television programs or magazines. Nakayama (2013) noted that many mothers experienced conflicts with other mothers regarding different aspects: different opinions regarding parenting practice (e.g., the child of my friend is not disciplined), concerns about the difference in socioeconomic status, and hassles such as "secrets" being leaked to others [78]. 
Hence, Japanese mothers, sensitively seeking a community network, regard starting new relationships with other mothers in the community as a big concern.

In dual-income families in Japan, work-life conflict is significantly associated with perinatal depression. Japanese society has not caught up with the rapidly increasing number of working mothers [79]. This is reflected by the shortage of nursery rooms, long working hours, and an unfriendly atmosphere in offices regarding both the mother and husband being able to take a vacation for childrearing. Husbands cannot take sufficient time to spend with their family due to an uncooperative working environment. It is still very rare for a husband to take parental leave from an office or company in Japan. Another factor is that husbands are not used to doing household chores because their generation grew up with stay-at-home mothers due to traditional gender roles.

\subsection{Preference of Male Infants, and Lower Socio-Economic Status in India}

As well as in China, Taiwan, Hong-Kong, Korea, and Turkey [80], in India boys are preferred to girls because boys can take over the family business as a leader, contributing to the economic prosperity of the family. In contrast, girls are often considered as a financial burden under a custom called the "Dowry system": where the family of bride should give a huge amount of money to the groom's family when they marry, because the bride is supposed to belong to groom's family and be financially supported after marriage. The dowry system is thought to put a great financial burden on the bride's family. In some cases, the dowry system leads to female infanticide in India [27]. In terms of gender preference, Japan is exceptional among Asian countries in that there is not such a preference for boys. Male preference is found to have gradually weakened over the past two decades, and instead, female preference is becoming more dominant. Moriizumi (2008) found that the majority of couples had a balanced gender preference towards daughters and sons [81]. A possible reason for daughter preference is that parents are expecting biological daughters to take care of them when they become older, rather than financial support.

It should be noted that lower socio-economic status in India is much more influential in perinatal depression than in Japan. India has achieved strong economic growth (Gross Domestic Product $=\$ 2.26$ trillion) contributing to a reduction in the poverty rate [82]. However, there is a big socio-economic gap that exists in some regions and social hierarchies called caste, which are regarded as a strong determinant of health in India, are present [83]. It is reported people belonging to scheduled castes (or scheduled tribes) are more likely to suffer from moderate/severe depression than others [84]. Social disadvantages such as lower education, poverty, and discrimination from others not only cause mental impairment, but also become an obstacle in access to care and treatment.

\subsection{Implications for Clinical Practice and Suggestions for Future Study}

Consistent with the previous literature review [4], many common factors with other countries were identified from this current review. There is ample literature on the psychological interventions for and prevention of perinatal depression [85-88]. Most of the reports were, however, from western countries. There has been a movement of Global Mental Health (GMH) to scale up evidence-based interventions in low-middle income countries, however, we should exercise caution when applying 'universal' prevention and interventions in culturally-different countries $[89,90]$. Socio-economic determinants regarding mental health may play a different role depending on each country. According to a previous study, which explored cross-country differences in the effects of socio-economic characteristics of patients with diabetes on subjective health-related wellbeing, clear differences were revealed in 15 countries [91]. Assari (2014) concluded each country should consider 'the context that shapes social and behavioral determinants' [91]. Considering these country-specific factors in our study, cultural adaptation of interventions or developing other culture-specific strategies is essential. Especially in India, enhancing social awareness of the importance of perinatal depression in family and community settings may be worth considering as a means of detecting at-risk populations for perinatal depression. Involving family members in a session is a well-known technique in interpersonal therapy [88] 
but this may be even more important in the prevention and treatment of perinatal depression in India. Involving not only the women themselves, but also family members such as mothers-in-law, in psychological counselling might be considered as a tool for helping deal with family issues, which is considered as an important correlate for perinatal depression. In Japan, community enhancement involving husbands, parents, and other social organizations, including the working office, companies, and nursing rooms, may be required. In particular, creating a parent-friendly working environment involving colleagues and employers of both the husband and wife, enhancing the social awareness of community members (e.g., family, parents, and colleagues) to have a new consensus that parents who both work and raise a child will be supported in a community, and establishing infrastructure such as nursery rooms, should be prioritized in order to deal with rapid changes in society.

\subsection{Limitations}

Although we included peer-reviewed papers written in English and Japanese and excluded those that had unclear quality, the studies retained in this review had varying statistical effects. However, we included studies that explored factors by means of univariate analysis in order to grasp a wider scope of potential cultural influences on the onset of perinatal depression. Additionally, we did not include Hindi-language articles because we assumed the number of relevant articles is small so far and we cannot assess the quality of these articles. In future studies, updating the findings to include Hindi-language articles may be required to capture broader evidence.

\section{Conclusions}

This literature review synthesized evidence regarding factors relating to perinatal depression in India and Japan. A poor relationship or conflict with the mother-in-law or other family members, and a preference for male infants were identified as specific to India. On the other hand, perinatal depression in Japanese women was characterized by conflict with the biological mother and relatives, concerns about social relationships with other mothers in the community, concerns about childrearing, and conflict with work-life balance. Understanding differences in the social and cultural backgrounds that relate to these factors is important in implementing culture-specific interventions, which may be needed as well as global standardized interventions. In India, involving the family in treatment could be effective, considering that interactions with family members is strongly associated with perinatal depression. In Japan, a community approach involving not only the family but also the working environment is essential for a mother-friendly society.

Supplementary Materials: The following are available online at www.mdpi.com/2227-9032/5/4/91/s1. Table S1: Checklist of Inclusion Criteria.

Acknowledgments: This work was supported by the Ministry of Education, Culture, Sports, Science, and Technology (MEXT)/Japan Science and Technology Agency (JST), under the Global Leadership Program (2014) of the University of Tokyo (140200000085). The authors would like to express utmost gratitude to Prof. Kenji Shibuya (the University of Tokyo), Mariko Gakiya and all the other members in the program, Vikram Patel (London School of Hygiene and Tropical Medicine), and all the members of NGO Sangath (Goa, India) for great supervision and support.

Author Contributions: M.T. and A.L. conceived and designed this systematic review. M.T., Y.O., and A.L. performed full-text reviews. Findings of reviews were discussed among all authors (M.T., Y.O., A.L., and T.K.) M.T. wrote the paper supervised by T.K.

Conflicts of Interest: The authors declare no conflict of interest.

\section{References}

1. Gavin, N.I.; Gaynes, B.N.; Lohr, K.N.; Meltzer-Brody, S.; Gartlehner, G.; Swinson, T. Perinatal depression: A systematic review of prevalence and incidence. Obstet. Gynaecol. 2005, 106, 1071-1083. [CrossRef] [PubMed]

2. American Psychiatric Association. Diagnostic and Statistical Manual of Mental Disorders, 5th ed.; American Psychiatric Association: Washington, DC, USA, 2013. 
3. Orsolini, L.; Valchera, A.; Vecchiotti, R.; Tomasetti, C.; Lasevoli, F.; Fornaro, M.; De Berardis, D.; Perna, G.; Pompili, M.; Bellantuono, C. Suicide during perinatal period: Epidemiology, risk factors, and clinical correlates. Front. Psychiatry 2016, 7, 138. [CrossRef] [PubMed]

4. Klainin, P.; Arthur, D.G. Postpartum depression in Asian cultures: A literature review. Int. J. Nurs. Stud. 2009, 46, 1355-1373. [CrossRef] [PubMed]

5. Norhayati, M.N.; Hazlina, N.H.; Asrenee, A.R.; Emilin, W.M. Magnitude and risk factors for postpartum symptoms: A literature review. J. Affect. Disord. 2015, 175, 34-52. [CrossRef] [PubMed]

6. Biaggi, A.; Conroy, S.; Pawlby, S.; Pariante, C.M. Identifying the women at risk of antenatal anxiety and depression: A systematic review. J. Affect. Disord. 2016, 191, 62-77. [CrossRef] [PubMed]

7. Iwata, H.; Mori, E.; Tsuchiya, M.; Sakajo, A.; Maehara, K.; Ozawa, H.; Morita, A.; Maekawa, T.; Aoki, K.; Tamakoshi, K. Predictors of depressive symptoms in older Japanese primiparas at 1 month post-partum: A risk-stratified analysis. Jpn. J. Nurs. Sci. 2016, 13, 147-155. [CrossRef] [PubMed]

8. Otake, Y.; Nakajima, S.; Uno, A.; Kato, S.; Sasaki, S.; Yoshioka, E.; Ikeno, T.; Kishi, R. Association between maternal antenatal depression and infant development: A hospital-based prospective cohort study. Environ. Health Prev. Med. 2014, 19, 30-45. [CrossRef] [PubMed]

9. Kitamura, T.; Yoshida, K.; Okano, T.; Kinoshita, K.; Hayashi, M.; Toyoda, N.; Ito, M.; Kudo, N.; Tada, K.; Kanazawa, K.; et al. Multicentre prospective study of perinatal depression in Japan: Incidence and correlates of antenatal and postnatal depression. Arch. Women's Ment. Health 2006, 9, 121-130. [CrossRef] [PubMed]

10. Patel, V.; Rodrigues, V.; DeSouza, N. Gender, poverty, and postnatal depression: A study of mothers in Goa, India. Am. J. Psychiatry 2002, 159, 43-47. [CrossRef] [PubMed]

11. The World Bank. Available online: https://data.worldbank.org/indicator/SP.DYN.TFRT.IN/ (accessed on 5 April 2017).

12. Chandra, P.S.; Venkatasubramanian, G.; Thomas, T. Infanticidal ideas and infanticidal behavior in Indian women with severe postpartum psychiatric disorders. J. Nerv. Ment. Dis. 2002, 190, 457-461. [CrossRef] [PubMed]

13. Shivalli, S.; Gururaj, N. Postnatal depression among rural women in South India: Do socio-demographic, obstetric and pregnancy outcome have a role to play? PLoS ONE 2015, 10, e0122079. [CrossRef] [PubMed]

14. Suzumiya, H.; Yamashita, H.; Yoshida, K. Hokenkikan ga jisshi suru boshihoumon taishousha no sango utu byou zenkoku tashisetu chousa. Kousei no Shihyou 2004, 51, 1-5. (In Japanese)

15. Vandenbroucke, J.P.; von Elm, E.; Altman, D.G.; Gøtzsche, P.C.; Mulrow, C.D.; Pocock, S.J.; Poole, C.; Schlesselman, J.J.; Egger, M.; STROBE Initiative. Strengthening the Reporting of Observational Studies in Epidemiology (STROBE): Explanation and elaboration. Epidemiology 2007, 18, 805-835. [CrossRef] [PubMed]

16. Sheela, C.N.; Venkatesh, S. Screening for postnatal depression in a tertiary care hospital. J. Obstet. Gynaecol. India 2016, 66, 72-76. [CrossRef] [PubMed]

17. Bodhare, T.N.; Sethi, P.; Bele, S.D.; Gayatri, D.; Vivekanand, A. Postnatal quality of life, depressive symptoms, and social support among women in southern India. Women Health 2015, 55, 353-365. [CrossRef] [PubMed]

18. Jain, A.; Tyagi, P.; Kaur, P.; Puliyel, J.; Sreenivas, V. Association of birth of girls with postnatal depression and exclusive breastfeeding: An observational study. BMJ Open 2014, 4, e003545. [CrossRef] [PubMed]

19. Srinivasan, N.; Murthy, S.; Singh, A.K.; Upadhyay, V.; Mohan, S.K.; Joshi, A. Assessment of burden of depression during pregnancy among pregnant women residing in rural setting of Chennai. J. Clin. Diagn. Res. 2015, 9, LC08-LC12. [CrossRef] [PubMed]

20. Johnson, A.R.; Edwin, S.; Joachim, N.; Mathew, G.; Ajay, S.; Joseph, B. Postnatal depression among women availing maternal health services in a rural hospital in South India. Pak. J. Med. Sci. 2015, 31, 408-413. [CrossRef] [PubMed]

21. Nongrum, R.; Thomas, E.; Lionel, J.; Jacob, K.S. Domestic violence as a risk factor for maternal depression and neonatal outcomes: A hospital-based cohort study. Indian J. Psychol. Med. 2014, 36, 179-181. [CrossRef]

22. Lukose, A.; Ramthal, A.; Thomas, T.; Bosch, R.; Kurpad, A.V.; Duggan, C.; Srinivasan, K. Nutritional factors associated with antenatal depressive symptoms in the early stage of pregnancy among urban South Indian women. Matern. Child Health J. 2014, 18, 161-170. [CrossRef] [PubMed]

23. Prost, A.; Lakshminarayana, R.; Nair, N.; Tripathy, P.; Copas, A.; Mahapatra, R.; Rash, S.; Gope, R.K.; Rath, S.; Bajpai, A.; et al. Predictors of maternal psychological distress in rural India: A cross-sectional community based study. J. Affect. Disord. 2012, 138, 277-286. [CrossRef] [PubMed] 
24. Dubey, C.; Gupta, N.; Bhasin, S.; Muthal, R.A.; Arora, R. Prevalence and associated risk factors for postpartum depression in women attending a tertiary hospital, Delhi, India. Int. J. Soc. Psychiatry 2012, 58, 577-580. [CrossRef] [PubMed]

25. Savarimuthu, R.J.; Ezhilarasu, P.; Charles, H.; Antonisamy, B.; Kurian, S.; Jacob, K.S. Post-partum depression in the community: A qualitative study from rural South India. Int. J. Soc. Psychiatry 2010, 56, 94-102. [CrossRef] [PubMed]

26. Varma, D.; Chandra, P.S.; Thomas, T. Intimate partner violence and sexual coercion among pregnant women in India: Relationship with depression and post-traumatic stress disorder. J. Affect. Disord. 2007, 102, 227-235. [CrossRef] [PubMed]

27. Rodrigues, M.; Patel, V.; Jaswal, S.; de Souza, N. Listening to mothers: Qualitative studies on motherhood and depression from Goa, India. Soc. Sci. Med. 2003, 57, 1797-1806. [CrossRef]

28. Chandran, M.; Tharyan, P.; Muliyil, J.; Abraham, S. Post-partum depression in a cohort of women from a rural area of Tamil Nadu, India. Incidence and risk factors. Br. J. Psychiatry 2002, 181, 499-504. [CrossRef] [PubMed]

29. Kita, S.; Haruna, M.; Matsuzaki, M.; Kamibeppu, K. Associations between intimate partner violence (IPV) during pregnancy, mother-to-infant bonding failure, and postnatal depressive symptoms. Arch. Women's Ment. Health 2016, 19, 623-634. [CrossRef] [PubMed]

30. Tachibana, Y.; Koizumi, T.; Takehara, K.; Kakee, N.; Tsujii, H.; Mori, R.; Inoue, E.; Ota, E.; Yoshida, K.; Kasai, K.; et al. Antenatal risk factors of postpartum depression at 20 weeks gestation in a Japanese sample: Psychosocial perspectives from a cohort study in Tokyo. PLoS ONE 2015, 10, e0142410. [CrossRef] [PubMed]

31. Shirakata, M.; Tanaka, Y.; Souke, K.; Okuyama, Y.; Fujii, H.; Takada, M.; Sawa, R.; Ebina, A.; Kondo, Y.; Ono, R. Ninshinki no yotu to nyou shikkin, utsubyou tono kanren. Hyogo J. Matern. Health 2014, 23, $20-22$. (In Japanese)

32. Fukao, S.; Kabeyama, K. Association between depressive tendency and stress-coping ability of pregnant and postpartum women following assisted reproductive technology and conventional infertility treatment: A longitudinal survey from late pregnancy to 1 month postpartum. Jpn. Acad. Midwifery 2014, 28, 260-267. (In Japanese) [CrossRef]

33. Amagai, S.; Emori, Y.; Murai, F.; Koizumi, J. Depression and life satisfaction in pregnant women: Associations with socio-economic status. Boseieisei 2014, 55, 387-395. (In Japanese)

34. Minatani, M.; Kita, S.; Ohashi, Y.; Kitamura, T.; Haruna, M.; Sakanashi, K.; Tanaka, T. Temperament, character, and depressive symptoms during pregnancy: A Study of a Japanese population. Depres. Res. Treat. 2013, 140169. [CrossRef] [PubMed]

35. Kinjo, H.; Yuge, M.; Kawasaki, K.; Takeo, K.; Kishi, K.; Lertsakornsiri, M.; Maleewan, L.; Takahashi, C.; Maruyama, Y. Comparative study on depression and related factors among pregnant and postpartum women in Japan and Thailand. Sakudaigaku Kango Kenkyu Zasshi 2013, 5, 5-19. (In Japanese)

36. Sugawara, S.; Ohira, H. The relationship between sense of coherence and mental health during the perinatal period. J. Child Health 2013, 72, 17-27. (In Japanese) [CrossRef]

37. Urayama, A.; Nagayama, K.; Ooki, H. Ninshincyu no jisonkanjyoutokuseitekijikokouryokukan tosango yokuutu tono kanrensei. Parinatal Care 2013, 32, 617-623.

38. Sugishita, Y.; Kamibeppu, K. Relationship between Prepartum and postpartum depression to use EPDS. Bosei Eisei 2013, 53, 444-450. (In Japanese)

39. Miyamoto, M. Mental health during pregnancy (part 1) - The correlation of depressive schemas with depressed mood in pregnant women. Boseieisei 2012, 52, 546-553. (In Japanese)

40. Nagasaka, I.; Sano, S. Research into factors during pregnancy for predicting postpartum depression: From the viewpoint of prevention of child abuse. J. Child Health 2012, 71, 737-747. (In Japanese)

41. Hayakawa, N.; Koide, T.; Okada, T.; Murase, S.; Aleksic, B.; Furumura, K.; Shiino, T.; Nakamura, Y.; Tamaji, A.; Ishikawa, N.; et al. The postpartum depressive state in relation to perceived rearing: A prospective cohort study. PLoS ONE 2012, 7, e50220. [CrossRef] [PubMed]

42. Kokubu, M.; Okano, T.; Sugiyama, T. Postnatal depression, maternal bonding failure, and negative attitudes towards pregnancy: A longitudinal study of pregnant women in Japan. Arch. Women's Ment. Health 2012, 15, 211-216. [CrossRef] [PubMed]

43. Kinjo, H.; Kawasaki, K.; Takeo, K.; Yuge, M.; Maruyama, Y.; Kishi, K. Depression symptoms and related factors on pregnant and postpartum women in Japan. Sakudaigaku Kango Kenkyu Zasshi 2012, 3, 15-25. 
44. Mori, T.; Tsuchiya, K.; Matsumoto, K.; Suzuki, K.; Mori, N.; Takei, N. Psychosocial risk factors for postpartum depression and their relation to timing of onset: The Hamamatsu Birth Cohort (HBC) Study. J. Affect. Disord. 2011, 135, 341-346. [CrossRef] [PubMed]

45. Miyake, Y.; Tanaka, K.; Sasaki, S.; Hirota, Y. Employment, income, and education and risk of postpartum depression: The Osaka Maternal and Child Health Study. J. Affect. Disord. 2011, 130, 133-137. [CrossRef] [PubMed]

46. Kikuchi, K.; Tomotake, M.; Iga, J.; Ueno, S.; Kahara, M.; Omori, T. Psychological features of pregnant women predisposing to depressive state during the perinatal period. J. Clin. Psychiatry 2010, 39, 1459-1468. (In Japanese)

47. Iwamoto, S.; Nakamura, M.; Yamashita, H.; Yoshida, K. Impact of situation of pregnancy on depressive symptom of women in perinatal period. J. Natl. Inst. Public Health 2010, 59, 51-59.

48. Arai, Y.; Takahashi, M. Family functioning and postpartum depression in women at one month postpartum. Kitasato Int. J. Nurs. Sci. 2009, 11, 1-9. (In Japanese)

49. Ando, T.; Muto, T. The course of depression from pregnancy through one year postpartum: Predictors and moderators. Jpn. J. Dev. Psychol. 2008, 23, 283-293. (In Japanese)

50. Sato, A.; Kitamiya, C.; Kudo, H.; Watanabe, M.; Menzawa, K.; Sasaki, H. Factors associated with late post-partum depression in Japan. Jpn. J. Nurs. Sci. 2009, 6, 27-36. (In Japanese) [CrossRef] [PubMed]

51. Kanazawa, R.; Narita, K.; Takahashi, H.; Kubato, C.; Yamada, A.; Godaigi, A.; Saga, M.; Akashi, Y. Edinburgh sango utubyou jikohyoukahyou kou tokutensha ni kyoutu suru haikei. Akita Nouson ikai shi 2008, 54, 30-34. (In Japanese)

52. Endo, K.; Nishiwaki, M.; Yamakawa, Y.; Komatsu, R.; Hori, M.; Tutumi, K.; Misawa, K.; Kawasaki, K. Predictors of postpartum depression in the early postpartum stage. Yamagata Hoken Iryou Kenkyu 2008, 11, 1-8. (In Japanese)

53. Mitamura, T.; Satou, H.; Mizukami, N. Risk factors of postpartum depression in low risk pregnant women. J. Jpn. Soc. Perinat. Neonatal Med. 2008, 44, 68-73.

54. Sekizuka, M.; Sakai, A.; Shimada, K.; Tabuchi, N.; Kameda, Y. Relationship between stress coping ability and the degree of satisfaction with delivery or postpartum depression tendency. Boseieisei 2017, 48, 106-113. (In Japanese)

55. Sato, N.; Morioka, Y.; Sato, A.; Oiji, A.; Murata, A. Relationships among mother's postpartum emotional state, her own attachment style and attachment formation toward her baby. Boseieisei 2006, 47, 320-329. (In Japanese)

56. Ninagawa, E.; Yamamoto, K.; Kawaguchi, N.; Tohi, T.; Yoshida, H.; Morinaga, H.; Kato, K.; Matsui, Y.; Nagamori, M.; Saito, M.; et al. Study on the Maternal Mental Health after Childbirth. Hokuriku J. Public Health 2005, 32, 45-48. (In Japanese)

57. Tokiwa, Y. Relationship between self-evaluation of child birth experience and early postpartum depression. J. Jpn. Acad. Midwifery 2003, 17, 27-38. (In Japanese) [CrossRef]

58. Iwatani, S.; Hokutou, H.; Wakabayashi, N.; Yoshikawa, T.; Naruse, E. The relation among the mental states at the early stage of pregnancy. J. Jpn. Soc. Psychosomatic Obstet. Gynaecol. 2001, 6, 116-123. (In Japanese) [CrossRef]

59. Tamaki, R.; Murata, M.; Okano, T. Risk factors for postpartum depression in Japan. Psychiatry Clin. Neurosci. 1997, 51, 93-98. (In Japanese) [CrossRef] [PubMed]

60. Cox, J.L.; Holden, J.M.; Sagovsky, R. Detection of postnatal depression: Development of the 10-item Edinburgh Postnatal Depression Scale. Br. J. Psychiatry 1987, 150, 782-786. [CrossRef] [PubMed]

61. Kroenke, K.; Spitzer, R.L.; Williams, J.B.W. The PHQ-9: Validity of a brief depression severity measure. J. Gen. Int. Med. 2001, 16, 606-613. [CrossRef]

62. Kessler, R.C.; Andrews, G.; Colpe, L.; Hiripi, E.; Mroczek, D.K.; Normand, S.L.; Walters, E.E.; Zaslavsky, A.M. Short screening scales to monitor population prevalences and trends in non-specific psychological distress. Psychol. Med. 2002, 32, 959-976. [CrossRef] [PubMed]

63. Beck, C.T. The effects of postpartum depression on child development: A meta-analysis. Arch. Psychiatry Nurs. 1998, 12, 12-20. [CrossRef]

64. Lewis, G.; Pelosi, A.J.; Araya, R.; Dunn, G. Measuring psychiatric disorder in the community: A standardized assessment for use by lay interviewers. Psychol. Med. 1992, 22, 465-486. [CrossRef] [PubMed]

65. Zung, W.W. A self-rating depression scale. Arch. Gen. Psychiatry 1965, 12, 63-70. [CrossRef] [PubMed] 
66. Zigmond, A.S.; Snaith, R.P. The hospital anxiety and depression scale. Acta Psychiatr. Scand. 1983, 67, 361-370. [CrossRef] [PubMed]

67. Radloff, L.S. The CES-D scale: A self-report depression scale for research in the general population. Appl. Psychol. Meas. 1977, 1, 385-401. [CrossRef]

68. First, M.B.; Spitzer, R.L.; Gibbon, M.; Williams, J.B.W. Structured Clinical Interview for DSM-IV-TR Axis I Disorders, Research Version, Patient Edition. (SCID-I/P); Biometrics Research, New York State Psychiatric Institute: New York, NY, USA, 2002.

69. Fernandes, M.C.; Srinivasan, K.; Stein, A.L.; Menezes, G.; Sumithra, R.; Ramchandani, P.G. Assessing prenatal depression in the rural developing world: A comparison of two screening measures. Arch. Women's Ment. Health 2011, 14, 209-216. [CrossRef] [PubMed]

70. Faisal-Cury, A.; Menezes, P.; Araya, R.; Zugaib, M. Common mental disorders during pregnancy: Prevalence and associated factors among low-income women in São Paulo, Brazil. Arch. Women's Ment. Health 2009, 12, 335-343. [CrossRef] [PubMed]

71. Golbasi, Z.; Kelleci, M.; Kisacik, G.; Cetin, A. Prevalence and correlates of depression in pregnancy among Turkish women. Matern. Child Health J. 2010, 14, 485-491. [CrossRef] [PubMed]

72. Okano, T.; Murata, M.; Masuji, S.; Tamaki, R.; Nomura, J.; Miyaoka, H.; Kitamura, T. Validity and reliability of Japanese Version of the EPDS (Edinburgh Postnatal Depression Scale). Arch. Psych. Diagn. Clin. Eval. 1996, 7, 525-533. (In Japanese)

73. Kitamura, T.; Shima, S.; Sugawara, M.; Toda, M.A. Temporal variation of validity of self-rating questionnaires: Repeated use of the General Health Questionnaire and Zung's Self-rating Depression Scale among women during antenatal and postnatal periods. Acta Psychiatry Scand. 1994, 90, 446-450. [CrossRef]

74. Kitayama, S.; Markus, H.R.; Matsumoto, H.; Norasakkunkit, V. Individual and collective processes in the construction of the self: Self-enhancement in the United States and self-criticism in Japan. J. Pers. Soc. Psychol. 1997, 72, 1245-1267. [CrossRef] [PubMed]

75. Corbett, C.A.; Callister, L.C. Giving birth: The voices of women in Tamil Nadu, India. MCN Am. J. Matern. Child Nurs. 2012, 37, 298-305. [CrossRef] [PubMed]

76. Harada, N. Statistical analysis of risk factors for depression of mothers with a high score on Edinburgh Postnatal Depression Scale. J. Health Sci. 2008, 5, 1-12. (In Japanese)

77. Minami, T.; Ohara, T.; Muto, Y. Childbirth and childcare for mother: Focus of experience of 'Childbirth Satogaeri'. J. Home Econ. Jpn. 2006, 57, 807-817. (In Japanese)

78. Nakayama, M.; Ikeda, Y. Relationship between interpersonal conflicts and personality traits in friendship between mothers rearing little children. Jpn. J. Pers. 2013, 22, 285-288. (In Japanese) [CrossRef]

79. Ministry of Labor and Health. 2016. Available online: http://www.mhlw.go.jp/file/05-Shingikai-11201000Roudoukijunkyoku-Soumuka/0000118655.pdf (accessed on 1 April 2017).

80. Xie, R.H.; He, G.; Koszycki, D.; Walker, M.; Wen, S.W. Fetal sex, social support, and postpartum depression. Can. J. Psychiatry 2009, 54, 750-756. [CrossRef] [PubMed]

81. Moriizumi, R. An empirical study of sex preferences for children in Japan. J. Popul. Probl. 2008, 64, 1-20. (In Japanese)

82. Organization for Economic Cooperation and Development (OECD). Available online: http:/ / www.oecd. org/eco/surveys/INDIA-2017-OECD-economic-survey-overview.pdf (accessed on 30 November 2017).

83. Kowal, P.; Afshar, S. Health and the Indian caste system. Lancet 2015, 385, 415-416. [CrossRef]

84. Shidhaye, R.; Lyngdoh, T.; Murhar, V.; Samudre, S.; Krafft, T. Predictors, help-seeking behaviour and treatment coverage for depression in adults in Sehore district, India. Br. J. Psychiatry Open 2017, 3, $212-222$. [CrossRef] [PubMed]

85. Austin, M.P.; Frilingos, M.; Lumley, J.; Hadzi-Pavlovic, D.; Roncolato, W.; Acand, S.; Saint, K.; Segal, N.; Parker, G. Brief antenatal cognitive behaviour therapy group intervention for the prevention of postnatal depression and anxiety: A randomized controlled trial. J. Affect. Disord. 2008, 105, 35-44. [CrossRef] [PubMed]

86. Brugha, T.S.; Morrell, C.J.; Slade, P.; Walters, S.J. Universal prevention of depression in women postnatally: Cluster randomized trial evidence in primary care. Psychol. Med. 2011, 41, 739-748. [CrossRef] [PubMed]

87. Zlotnick, C.T.; Miller, I.W.; Pearlstein, T.; Howard, M.; Sweeney, P. A preventive intervention for pregnant women on public assistance at risk for postpartum depression. Am. J. Psychiatry 2006, 163, 1443-1445. [CrossRef] [PubMed] 
88. Weissman, M.M.; Markowitz, J.C.; Klerman, G. Comprehensive Guide to Interpersonal Psychotherapy; Basic Books: NewYork, NY, USA, 2000.

89. White, R.G.; Sashidharan, S.P. Towards a more nuanced global mental health. Br. J. Psychiatry 2014, 204, 415-417. [CrossRef] [PubMed]

90. Jain, S.; Orr, D.M. Ethnographic perspectives on global mental health. Transcult. Psychiatry 2016, 53, 685-695. [CrossRef] [PubMed]

91. Assari, S. Cross-country variation in additive effects of socio-economics, health behaviors, and comorbidities on subjective health of patients with diabetes. J. Diabetes Metab. Disord. 2014, 13, 36. [CrossRef] [PubMed] 